\title{
La radioprotection au quotidien dans une centrale nucléaire
}

\author{
(Manuscrit reçu le 5 mai 1988) \\ J.-L. VIOT*
}

\begin{abstract}
RÉSUMÉ
L'article présente quelques-uns des temps forts du travail effectué par la Section sécurité radioprotection de la Sous-unité 3-4 du Centre de production nucléaire de Dampierre, au cours des dernières années. Les actions décrites illustrent les efforts engagés sur le terrain pour réduire l'exposition des travailleurs, qu'il s'agisse de l'irradiation externe ou interne. Au-delà des exemples, l'article souligne également les principes selon lesquels est menée l'activité de la section : enquêtes et analyses à la suite de l'identification de situations anormales, préparation et suivi des chantiers pour les opérations importantes de maintenance. Quelques propositions sont suggérées en conclusion qui pourraient encore améliorer l'efficacité de la radioprotection dans les centrales.
\end{abstract}

\section{ABSTRACT}

The paper presents some of the salient works performed during these last years by the Radiological protection and security section of Units 3 and 4 of the Dampierre Nuclear power station. The actions described are illustrating some of the day-to-day efforts to reduce occupational exposures for both external irradiation and internal contamination. Beyond the examples, the paper outlines the basic principles structuring the section's activity: investigations and analysis in case of abnormal situations, preparation and follow-up of large maintenance operations. Some propositions are addressed in conclusion to further improve the occupational radiological protection in nuclear power stations.

\section{INTRODUCTION}

Au-delà des tâches immédiates et concrètes concernant la préparation et l'entretien du matériel de radioprotection, l'information et la formation du personnel, la dosimétrie, les agents des sections "sécurité radioprotection" (SRP) sont, en permanence, confrontés à des problèmes dont les solutions ne sont, a priori, pas évidentes.

* Section SRP, Centrale 3-4, Centre de production nucléaire de Dampierre, BP 18, 45570 Ouzouer-sur-Loire. 
Le travail d'enquête et d'analyse, la mise au point de mesures de protection ou d'actions correctives, font partie des missions qui doivent contribuer à l'amélioration de la radioprotection des travailleurs.

Les développements qui suivent présentent quelques-uns des temps forts de la SRP de la sous-unité 3-4 du Centre de production nucléaire de Dampierre. Les exemples retenus montrent comment cette action se développe et s'organise en relation avec les services centraux d'Electricité de France. L'article présente les enseignements que l'on peut tirer de l'expérience récente et propose quelques mesures simples qui pourraient contribuer à l'amélioration de la qualité de la radioprotection dans les centrales.

\section{LE TRAVAIL D'ENQUETE ET D'ANALYSE}

A l'occasion des travaux d'exploitation et de maintenance, une part importante de l'activité de la SRP est consacrée à la mesure des débits de dose ambiants et des niveaux de contamination atmosphérique dans les locaux où les opérateurs doivent intervenir. La détection de niveaux d'exposition anormalement élevés conduit systématiquement à une enquête pour en déterminer les causes et proposer, après analyse du problème, des solutions techniques pour rétablir la situation et mieux contrôler les sources.

Ce travail d'enquête et d'analyse est, dans la plupart des cas, effectué au sein de la section et les actions correctives sont engagées au niveau de la centrale. Cependant, pour certains problèmes complexes, qui peuvent présenter par ailleurs un caractère générique pour l'ensemble des tranches du parc, la SRP informe et, parfois, travaille avec le Département "sécurité, radioprotection, environnement" (DSRE) du Service de la production thermique.

Les paragraphes suivants décrivent quelques exemples de problèmes qui ont été soulevés par la SRP de Dampierre et qui ont débouché sur l'amélioration d'équipements ou de procédures au niveau de la centrale ou qui ont été le point de départ d'une action de recherche et développement au niveau des services centraux.

\subsection{Le contrôle des sources}

\subsubsection{Sources responsables d'irradiations externes}

Un débit de dose inhabituel (près de $0,1 \mathrm{~Sv} / \mathrm{h}$ au contact) est relevé en août 1984 sur une pompe du circuit de contrôle volumétrique et chimique (RCV) lors de l'arrêt pour rechargement du combustible de la tranche 3 . Des frottis réalisés à la suite de ces mesures montrent que plus de $99 \%$ de l'activité déposée sur des éléments de cette pompe est due à l'argent 110 métastable $\left({ }^{110 \mathrm{~m}} \mathrm{Ag}\right)$. Existe-t-il dans tous les circuits? A quel moment apparaît-il? D'où vient-il? Quelle est sa part de responsabilité dans la dosimétrie d'un arrêt de tranche? Telles sont les questions qui se posent à la SRP. 
En poursuivant les investigations, on découvre que ${ }^{110 \mathrm{~m}} \mathrm{Ag}$ se dépose essentiellement dans le circuit de contrôle volumétrique et chimique (RCV), qui permet de réguler la quantité et la qualité de l'eau du circuit primaire, où son taux varie de 65 à $99 \%$, alors que dans le circuit primaire lui-même, son taux reste inférieur à $1 \%$. L'examen des relevés journaliers du système de surveillance d'activité du circuit RCV révèle des comptages plus importants au moment de l'arrêt à froid de mai 1985 - arrêt spécifique pour bouchages de tubes de générateur de vapeur - puis leur diminution très faible mais régulière. Les analyses radiochimiques effectuées le 12 mai 1985 présentent un pic de ${ }^{110 \mathrm{~m}} \mathrm{Ag}$ de $904 \mathrm{MBq} /$ tonne, pic situé après la phase d'oxygénation du circuit primaire.

Malgré ces éléments nouveaux, la question de l'origine de ${ }^{110 \mathrm{~m}} \mathrm{Ag}$ reste posée. La section fait appel au DSRE et, après réflexion, les recherches s'orientent du côté des crayons de la grappe de commande du réacteur. En effet, cette dernière, qui est l'organe mécanique de réglage de la réaction en chaîne de la fission par insertion dans le cœur d'un matériaux absorbant neutronique, est constituée de crayons (2 à 2,4 tonnes) qui sont composés à $80 \%$ d'argent, $15 \%$ d'indium et $5 \%$ de cadmium. Les énergies sont mobilisées pour l'arrêt suivant qui doit avoir lieu sur la tranche 4 en juin 1985. Le service "chimie" prouve, par voie chimique et dosage par spectrométrie d'absorption atomique, la présence de traces de cadmium. Le service "essais", par l'inspection télévisuelle de 8 grappes sur le dernier mètre de leur partie basse, met en évidence des traces d'usures au niveau des pas de fonctionnement des grappes.

Parallèlement à cette action, le Département matériel du Service de la production thermique, informé par le DSRE du problème de ${ }^{110 \mathrm{~m}} \mathrm{Ag}$ et de son origine possible au niveau des grappes, entreprend sur la tranche 4 de la centrale de Tricastin une inspection poussée des grappes par courant de Foucault, ultrasons et télévisualisation. Le bilan de cette inspection, publié en avril 1986, met en lumière:

- que l'usure est au niveau du guidage continu et au niveau des cartes de guidage;

- que toutes les grappes ont, à des degrés divers, plusieurs crayons affectés;

- que les usures au droit des cartes de guidage sont les plus importantes au point que 18 grappes ont au moins un crayon fortement affecté. La profondeur d'usure, dans ce cas, est supérieure à $0,30 \mathrm{~mm}$ pour une épaisseur de gaine de $0,47 \mathrm{~mm}$. Deux crayons sont percés.

En octobre 1986, un premier programme est lancé. Outre des études techniques portant sur les critères de limites d'usure des crayons, sur l'état des grappes du parc des tranches à eau sous pression de $900 \mathrm{MW}$, sur de nouvelles conceptions de grappes, des dispositions conservatoires sont prises pour les grappes existantes (décalage de trois pas, permutations...) et des stratégies sont élaborées prévoyant à terme le remplacement des grappes. Une modification des structures internes supérieures situées dans la cuve au-dessus des éléments combustibles n'est pas écartée. 
Ces développements ne sont plus du ressort de la SRP, mais cependant cette dernière reste mobilisée, car si les premières estimations de dose imputables à ${ }^{110 \mathrm{~m}} \mathrm{Ag}$ étaient évaluées en avril 1985 à $10 \%$ de la dosimétrie d'un arrêt de tranche, rien n'indique qu'il s'agit d'une valeur définitive. Des mesures effectuées lors de l'arrêt de la tranche 1 de Dampierre ont révélé des débits de dose importants au contact du circuit RCV $(0,1$ à $0,25 \mathrm{~Sv} / \mathrm{h})$; l'ambiance dans le local de l'échangeur du RCV 002 RF étant même montée à $25 \mathrm{mSv} / \mathrm{h}$. Dans l'attente d'une résolution du problème, la SRP reste vigilante et prend des mesures d'adaptation: mise en place de protections biologiques et décontamination d'une portion du circuit RCV par chimie douce (cette dernière étant réalisée en collaboration avec l'entreprise Gagneraud).

\subsubsection{Sources à potentiel d'irradiation interne}

Contamination de locaux hors zone contrôlée. Lors du redémarrage de la tranche 3 en octobre 1984, une soupape non remontée sur le circuit RCV provoque, par des inétanchéités de passages de tuyauteries et de trémies de câble, une inondation de locaux hors zone contrôlée. Des effluents arrivent jusqu'à un puisard de la galerie centrale où une activité gamma totale de $62,2 \mathrm{MBq} / \mathrm{m}^{3}$ est décelée. Circonscrire les zones contaminées, contrôler la décontamination, rechercher les origines matérielles prendront deux mois. Réaliser tous les travaux demanderont deux ans.

Depuis, la section reste vigilante car les améliorations et modifications apportées en centrale nucléaire vont généralement de pair avec de nouveaux tirages de câbles. II est important que les locaux extérieurs à la zone restent à l'abri de toute contamination: un "no man's land" doit subsister entre la "zone contrôlée" et la nappe phréatique. Céder à la facilité n'est pas de mise.

Transfert de contamination volumique. Dans le bâtiment de stockage du combustible, une balise de surveillance de la contamination passe en alarme rouge, le personnel est évacué... Lors d'essais périodiques, des agents du service exploitation sont régulièrement déclarés "contaminés" aux portiques C2 de sortie de zone contrôlée... Lors de l'arrêt de tranche 3 , une évacuation du bâtiment réacteur est lancée à la suite d'une alarme apparue sur la chaîne de surveillance de l'activité de l'air de l'enceinte.

L'analyse de ces trois cas par la section montre que c'est le système de réseau de collecte des purges et des évents (circuits RPE) qui est en cause. Lorsque ce dernier reçoit les effluents liquides radioactifs, il en disperse les effluents gazeux.

Pour stopper cette situation, une garde hydraulique est installée sur le trop-plein de la piscine du bâtiment combustible de la tranche impaire, des bouchages d'entonnoirs sont réalisés en attendant l'installation de vannes sur des portions du circuit RPE. II reste à traiter, dans le cadre d'un groupe de travail, la nécessaire mise en dépression de ce circuit RPE et la prise en compte du risque iode dans les locaux non classés pour ce risque. 
II serait possible d'évoquer d'autres exemples sur lesquels a travaillé la section SRP (iode, émetteurs alpha...) et qui participent au même objectif de non irradiation interne des agents EDF et des entreprises prestataires. Cependant, la section n'est pas seulement une section d'analyse: elle contribue aussi directement à l'amélioration des équipements.

\subsection{L'amélioration des équipements}

L'identification de sources "anormales" d'exposition ou de tâches inutiles, et le développement de mesures correctives après analyse de la situation, sont des objectifs permanents de la section SRP. Les actions décrites dans ce paragraphe donnent une idée du type d'activité lors d'un arrêt de tranche pour rechargement du combustible.

La mise en place de protections biologiques. Lors de manutentions du combustible, à l'occasion d'un déchargement de réacteur, un débit de dose anormalement élevé est détecté dans l'espace annulaire du bâtiment réacteur au niveau du "tube transfert" entre la piscine du réacteur et la face interne de l'enceinte du bâtiment réacteur: plus de 0,75 Sv/h est mesuré au niveau $11 \mathrm{~m}$ le long de l'enceinte et ce malgré les protections déjà installées. Dans un premier temps, un système de balisage avec des "franchissements interdits" est mis en place pour éviter des expositions inutiles. II s'avère, cependant, que ce balisage est source d'allées et venues importantes et donc de pertes de temps. Après analyse du problème, une protection biologique complémentaire est installée autour du tube de transfert. Cette protection en forme de tunnel est constituée de 3 épaisseurs de briques de plomb à chevron avec une chicane d'accès dont l'ouverture donne du côté piscine du bâtiment réacteur. Les "fuites" sont ainsi réduites à zéro.

La modification des installations. A l'occasion des travaux dans les générateurs de vapeur qui doivent être effectués en tenue étanche ventilée du fait du risque de contamination, les gardiens des sas de confinement sont régulièrement sollicités pour déméler les "boyaux" d'alimentation en air comprimée des tenues qui, d'une longueur de $12,5 \mathrm{~m}$, s'emmèlent constament dans ces sas. Pour remédier à ces interventions coûteuses en doses, des barillets de distribution d'air sont installés à demeure au niveau $8 \mathrm{~m}$ de chaque générateur de vapeur permettant de réduire la longueur des "boyaux" à $6 \mathrm{~m}$.

Une innovation brevetée. La dépose du couvercle de la cuve du réacteur, lors des ouvertures de cuve pour rechargement du combustible, est une opération délicate sur le plan de la radioprotection, tant du point de vue des irradiations que des risques de contamination. Lorsque le couvercle est sur son stand de dépose, il existe en effet une "ligne de fuite" entre le couvercle et l'anneau en béton sur lequel il repose. Par ailleurs, les risques de contamination obligent à isoler le couvercle par une paroi en vinyl "scotchée", et à installer un toit de sas de confinement au-dessus du couvercle. Afin de réduire les doses associées aux diverses opérations, des mesures sont prises. Une armature en quatre éléments repliables, qui est à demeure dans le bâtiment réacteur, permet d'installer, en $10 \mathrm{~min}$, une paroi vinyle recyclable (fruit d'une collaboration SRP-SOGEDEC). La mise en 
œuvre du confinement dynamique permet de supprimer le toit de sas et de réduire au maximum le port de tenues ventilées. Les mêmes dispositions qu'au niveau $8 \mathrm{~m}$ du générateur de vapeur permettent de réduire les temps de séjour liés à l'emmêlement des boyaux. Enfin, une couronne de plomb de $5 \mathrm{~cm}$ d'épaisseur et de $30 \mathrm{~cm}$ de hauteur est installée au-dessus de l'anneau, ce qui fait chuter le débit de dose de la ligne de fuite d'un facteur 3 à 4 .

II est intéressant de noter que le système de confinement dynamique inventé par deux agents SRP a donné lieu au dépot d'un brevet avec l'aide du groupe de la Promotion des applications de la recherche dans l'industrie (EDF). Son application industrielle sera lancée par l'Unité technique opérationnelle (UTO) du Service de la production technique et deux ensembles seront disponibles par centrale.

II serait possible d'évoquer d'autres exemples, qui font appel à l'esprit d'analyse et de déduction ainsi qu'au sens de l'innovation, et qui participent à l'amélioration constante de la radioprotection. Cependant, au cours de ces dernières années, c'est dans le domaine de la radioprotection des grands chantiers de maintenance que les agents des SRP se sont trouvés confrontés à de nouveaux problèmes nécessitant une certaine remise en cause de leur pratique.

\section{LA PRÉparation, Le Suivi et L'ANALyse des Chantiers}

II est bon de rappeler qu'environ $80 \%$ des expositions sont reçues en période d'arrêt de tranche et qu'au fil des années, avec le vieillissement des installations, la part des doses reçues à l'occasion des chantiers de maintenance dite exceptionnelle (réparation d'équipements, remplacement de matériels usés...) tendra à prendre le pas sur celle associée à la maintenance de routine. Dans ce contexte, il est apparu important à la section radioprotection de préparer avec une attention particulière les opérations exceptionnelles.

Cette préparation n'a évidemment d'intérêt que si, par ailleurs, les agents de la radioprotection se donnent les moyens de suivre attentivement les chantiers afin d'en tirer les enseignements pour les futurs chantiers identiques qui interviendront à l'occasion d'arrêts ultérieurs, soit sur d'autres tranches de la centrale, soit dans d'autres centrales. L'action menée par la SRP à l'occasion des opérations de microbillage des tubes des générateurs de vapeur effectuées sur les tranches 3 et 4 de la centrale de Dampierre en octobre 1985 et en mai 1986 illustrent comment la préparation, le suivi et l'analyse du retour d'expérience constituent les étapes indispensables pour assurer la réduction des expositions lors des grands chantiers de maintenance. De cette expérience, il est possible de tirer un certain nombre d'enseignements qui peuvent orienter la radioprotection de terrain pour les années à venir. 


\subsection{Le microbillage de Dampierre 3 (octobre 1985)}

Cette opération, qui était la seconde de ce type réalisée en France, a été effectuée lors de l'arrêt pour rechargement de la tranche 3 . En prenant connaissance de la dose totale engagée lors de la première expérience menée à la centrale de Doel en Belgique (0,93 homme-sievert), la SRP essaie d'obtenir des renseignements auprès des agents EDF qui viennent d'effectuer la première opération en France. A part quelques détails et beaucoup de généralités, nous n'arrivons pas à savoir dans quels locaux se prennent les doses au cours du chantier, quels sont les principaux problèmes rencontrés avec les porteurs d'outils (voir encadré) qui doivent être installés dans les boîtes à eau des générateurs de vapeur...

\section{Les deux porteurs d'outils disponibles à l'époque du microbillage de Dam-} pierre 3 étaient I'“Araignée" et le bras dit " $R$ têta" (voir figures).

- La mise en place de I'“Araignée" demande $24 \mathrm{~h}$ de préparation. Entre 120 et 130 tubes de générateur de vapeur peuvent être microbillés par poste de $8 \mathrm{~h}$. Bien que I'“Araignée" se déplace automatiquement d'un tube à l'autre, pour traiter l'ensemble des tubes dans une boîte à eau, il est cependant nécessaire de pénétrer 6 fois à l'intérieur de la boîte afin d'effectuer manuellement des rotations de I'“Araignée".

- La mise en place du bras " $R$ têta" demande $48 \mathrm{~h}$ de préparation comprenant, entre autre, le soudage de son axe vertical au fond du "bol" du générateur de vapeur. Entre 140 et 150 tubes peuvent être traités par poste. Les 60 derniers tubes qui correspondent à l'encombrement de l'axe du bras doivent être microbillés à l'aide de I'“Araignée".

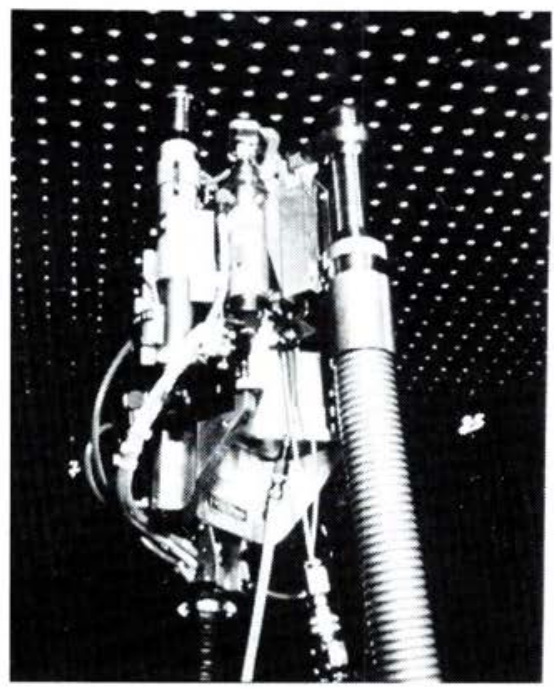

"Araignée".

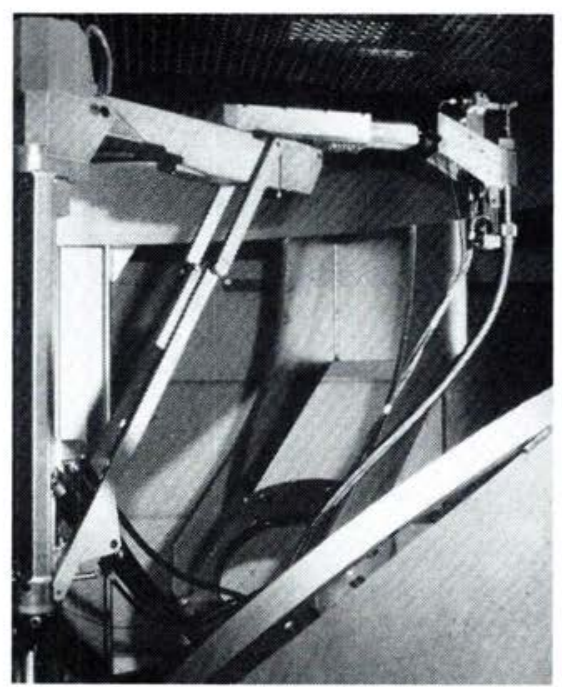

Bras "R têta". 
A Dampierre, où le microbillage doit être effectué sur deux générateurs de vapeur avec le bras "R têta" et sur le troisième au moyen de l'“Araignée", nous ne savons pas quel est le système le plus avantageux dans la mesure où le bras "R têta", a priori plus intéressant, est encore dans sa phase de mise au point.

Malgrè les incertitudes, quelques initiatives sont prises pour mieux maîtriser le chantier sur le plan de la radioprotection. Des fûts en béton sont préparés au niveau $4 \mathrm{~m}$ dans le bâtiment réacteur pour recevoir les microbilles usées très contaminées.

$\mathrm{Vu}$ les risques d'irradiation et de contamination, tous les sas de confinement, qu'ils soient sur la plate-forme $8 \mathrm{~m}$ de chaque générateur de vapeur ou qu'ils soient à $4 \mathrm{~m}$ sous chaque générateur, disposent d'un gardien. Celui-ci est, en particulier, chargé d'enregistrer sur un "cahier de sas" la dosimétrie de chaque intervenant à l'entrée et à la sortie des sas ainsi que la durée et le type de chaque intervention...

Par ailleurs, l'entreprise FRAMATOME, qui est chargée du chantier, met en place de son côté, sur deux générateurs, un système de recyclage automatique des microbilles qui doit permettre de supprimer les doses associées au recyclage manuel des microbilles. Au cours du chantier, la SRP intervient en particulier pour mettre en place des protections biologiques directement sur les organes les plus actifs. II convient, cependant, de noter qu'une réelle protection entraîne souvent soit une gêne, soit un risque d'accident.

Au total, et malgré ces précautions, le bilan dosimétrique de l'opération reste lourd: 0,8 homme-sievert dont 0,67 pour les agents FRAMATOME. L'analyse de cette dosimétrie et des mesures de débit de dose effectuées régulièrement au cours du chantier permet, cependant, de tirer plusieurs enseignements importants.

Le choix du système porteur. Les résultats (tableau I) conduisent la SRP à remettre en question l'utilisation de deux bras " $R$ têta". En effet, la plupart des problèmes techniques rencontrés par les agents FRAMATOME, qui ont contribué pour $40 \%$ de leur dosimétrie, l'ont été sur les générateurs microbillés avec les bras. Lors d'une réunion de bilan en décembre 1985 à l'Unité technique opérationnelle (UTO) du Service de la production thermique (I'UTO est chargée du suivi technique de la campagne de microbillages), la SRP propose de travailler désormais avec deux araignées et un bras, escomptant ainsi une réduction de l'exposition collective d'environ 0,10 homme-sievert.

TABLEAU I

Exposition collective associée au microbillage des générateurs de vapeur de Dampierre 3 en fonction de la technique utilisée.

\begin{tabular}{|c|c|c|}
\hline Générateur de vapeur & Type de porteur et de recyclage & Exposition collective \\
\hline$N^{\circ} 1$ & "R têta" + automatique & 0,336 homme-Sv \\
$N^{\circ} 2$ & "Araignée" + automatique & 0,108 homme-Sv \\
$N^{\circ} 3$ & "R têta" + manuel & 0,227 homme-Sv \\
\hline
\end{tabular}


L'adaptation du matériel. Les mesures de débit de dose montrent que l'ambiance dans le sas de confinement au niveau $8 \mathrm{~m}$ (plate-forme du générateur de vapeur) passe d'environ $0,3 \mathrm{mSv} / \mathrm{h}$ avant l'opération de microbillage à $2 \mathrm{mSv} / \mathrm{h}$ en fin d'intervention. II s'avère que les annelures intérieures des "boas" (qui permettent la circulation des microbilles entre les divers équipements) retiennent partiellement les microbilles d'où des débits au contact de ces derniers de l'ordre de 30 à $50 \mathrm{mSv} / \mathrm{h}$. La SRP suggère donc à I'UTO d'utiliser des "boas" lisses intérieurement.

L'introduction du recyclage automatique. L'analyse détaillée de la dosimétrie montre qu'il a permis un gain d'environ 0,25 homme-Sv par générateur de vapeur.

La distribution des doses individuelles. Près de $85 \%$ de l'exposition collective résulte de doses individuelles journalières inférieures à $1 \mathrm{mSv}$. La moyenne des doses cumulées des agents FRAMATOME reste cependant élevée: $7,4 \mathrm{mSv}$. La dose individuelle cumulée la plus importante est de $16,22 \mathrm{mSv}$.

\subsection{Le microbillage de Dampierre 4 (mai 1986)}

Pour cette deuxième opération, plusieurs mois avant le début du chantier cette fois, des contacts sont pris avec le responsable FRAMATOME du microbillage des générateurs de la tranche 4 pour préparer le chantier. Après examen de la situation, il est décidé, entre autre, d'utiliser le seul bras "R têta" au niveau du générateur $n^{\circ} 3$. En effet, compte tenu de l'expérience précédente, le bras semble le mieux adapté pour ce générateur de vapeur qui présente le plus grand nombre de tubes bouchés et un débit de dose supérieur de 10 à $20 \mathrm{mSv} / \mathrm{h}$ par rapport aux deux autres générateurs. Ces contacts permettent par ailleurs de mettre au point un système de protections biologiques au niveau des filtres et des vannes qui, à $4 \mathrm{~m}$ sous les générateurs de vapeur, peuvent atteindre des débits de dose importants $(0,25 \mathrm{~Sv} / \mathrm{h}$ à Dampierre 3$)$. En conséquence, la SRP fait construire des armatures métalliques pour accrocher des matelas de billes de plomb autour de ces filtres.

Au cours du chantier, l'évolution des débits de dose au niveau de chaque filtre est suivie journellement ainsi que celle des débits d'ambiance dans les sas de confinement. Ces dernières mesures montrent une évolution préoccupante dans les sas au niveau $8 \mathrm{~m}$ au fur et à mesure du déroulement du chantier, évolution particulièrement nette sur le générateur $\mathrm{n}^{\circ} 1$ de fabrication Vallourec, la SRP entreprend alors une investigation systématique des divers composants des équipements de microbillage dans les sas de confinement. Cette recherche conduit à isoler deux sources prépondérantes: le $\mathrm{Y}$ du tireur-pousseur (ce dernier permet les déplacements de l'outil de microbillage) et les "boas" de retour des microbilles. Des protections biologiques sont alors mises en place à l'aide de supports appropriés et les débits ambiants sont réduits de moitié. 
L'analyse de ce deuxième chantier montre que le bilan dosimétrique s'est nettement amélioré puisque l'exposition collective totale ne s'élève qu'à 0,39 homme-Sv dont 0,32 pour les opérateurs FRAMATOME, soit une réduction de l'ordre de $50 \%$ par rapport à l'opération de Dampierre 3 . De plus, elle permet de tirer d'autres enseignements parmi lesquels on peut mettre en avant:

Le choix du système porteur. La présence des concepteurs du bras " $R$ têta" a contribué à ce que le bras devienne un concurrent sérieux de I'“Araignée" (voir tableau II). Le coût dosimétrique des aléas, très faible $(0,04$ homme-sievert), malgré le démontage d'un bras pour nettoyage consécutif à un empoussiérage excessif, est dû à $85 \%$ aux "Araignées".

\section{TABLEAU ॥}

Exposition collective associée au microbillage des générateurs de vapeur de Dampierre 4 en fonction de la technique utilisée.

\begin{tabular}{|c|c|c|}
\hline Générateur de vapeur & Type de porteur et de recyclage & Exposition collective \\
\hline$N^{\circ} 1$ & "Araignée" + automatique & 0,125 homme-Sv \\
$N^{\circ} 2$ & "Araignée" + automatique & 0,103 homme-Sv \\
$N^{\circ} 3$ & "R têta" + automatique & 0,096 homme-Sv \\
\hline
\end{tabular}

La distribution des doses individuelles. La dose individuelle maximale s'élève à 13,23 mSv. La dose individuelle moyenne des agents FRAMATOME est, cette fois, de $4,87 \mathrm{mSv}$. La comparaison avec les résultats de Dampierre 3 (tableau III) montre que plus de $53 \%$ de ces agents ont reçu une dose inférieure à $5 \mathrm{mSv}$ contre $14 \%$ seulement lors de l'opération sur la tranche 3 , et que $5 \%$ ont reçu plus de $10 \mathrm{mSv}$ contre presque $37 \%$ à Dampierre 3 . Ces résultats démontrent que la réduction des doses individuelles peut aller de pair avec celle des expositions collectives.

TABLEAU III

Distribution des doses individuelles des agents FRAMATOME pour les microbillages de Dampierre 3 et 4 .

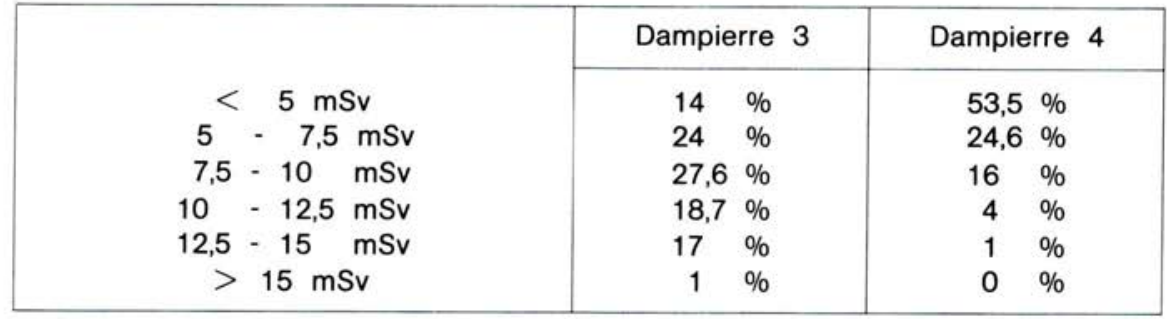


Le repli du chantier. Le bilan de cette phase, bien que similaire à celui du chantier précédent, apparaît cette fois relativement lourd $(0,083$ homme-sievert soit $25 \%$ de la dose totale) du fait de la réduction des expositions associées aux autres phases de l'opération. Cette constatation démontre que des progrès sont encore possibles au niveau de l'organisation du travail dans la phase de repli.

A la suite du chantier, une réunion de bilan est organisée entre les responsables de FRAMATOME et les agents de la SRP ayant suivi l'opération. Cette réunion permet de dresser une liste de mesures pour améliorer la dosimétrie des futurs microbillages. Parmi ces mesures, plusieurs sont relatives aux équipements qui peuvent être modifiés pour faciliter leur manutention et isoler les sources principales de débits de doses comme le remplacement du système de boas, l'installation de crochets de levage sur les matériels pour les transférer à l'aide d'un pont roulant... Par ailleurs, l'accent est porté sur les aspects d'organisation du travail afin d'éviter les tâches inutiles. Compte tenu de ces suggestions, un objectif de 0,2 à 0,3 homme-sievert est envisagé pour les chantiers suivants (l'expérience acquise en 1987 a montré que cet objectif était tout à fait réaliste).

\section{CONCLUSIONS}

Les exemples décrits montrent que la maîtrise des expositions dans les centrales passe par une recherche systématique des sources, des équipements et des travaux élémentaires qui sont à l'origine des doses. Cette recherche s'appuie essentiellement sur les mesures de débits de dose et de contamination dans tous les locaux où doivent intervenir les opérateurs, mais également sur l'analyse détaillée des doses individuelles associées aux tâches élémentaires effectuées par les opérateurs.

Compte tenu de l'ampleur du parc de réacteurs français et du haut niveau de standardisation des tranches, pratiquement toutes les opérations sont appelées à être répétées de nombreuses fois et toutes les anomalies repérées sur une tranche ont également de fortes chances d'apparaître sur les autres. L'expérience de ces dernières années pousse la radioprotection à accélérer le retour d'expérience pour atteindre les objectifs de la section: réduire de manière efficace les irradiations externes et supprimer les irradiations internes.

De cette expérience, nous dégageons quelques axes essentiels:

- intégrer les responsables SRP des centrales dans la préparation des programmes des chantiers répétitifs;

- favoriser le retour d'expérience pour ces chantiers répétitifs en faisant participer systématiquement un agent SRP du chantier $\mathrm{N}$ aux trois phases du déroulement du chantier $\mathrm{N}-1$ (préparation, suivi et analyse);

- fixer des objectifs dosimétriques au fur et à mesure de la réalisation d'un programme; 
- développer des outils analytiques pour améliorer le recueil des informations dosimétriques au plus près des chantiers et ne pas se contenter des informations récupérées en sortie de zone contrôlée;

- coordonner la répartition des tâches de radioprotection entre les entreprises prestataires et les agents SRP des centrales;

- intégrer le retour d'expérience de manière plus réactive en développant la constitution de groupes de travail pour tous les grands programmes de maintenance avec la participation des principaux intervenants: DSRE, UTO et les diverses SRP des centrales concernées.

En fait, il s'agit essentiellement, pour assurer cette "radioprotection réactive", de favoriser la circulation des informations entre les divers protagonistes. A ce niveau, il serait intéressant d'envisager la création d'un journal périodique inter-SRP qui pourrait traiter de tous les événements, les réalisations, les expériences des agents de terrain et ce, aussi bien dans le domaine de la radioprotection que dans celui de la sécurité classique. Parallèlement à cette initiative, il serait également souhaitable d'organiser des réunions inter-SRP pour approfondir certains problèmes difficiles. Dans le cadre de ces réalisations, le DSRE pourrait avoir un rôle nouveau d'écoute et d'animation. 